\title{
REFORMANDO EL SECTOR ELÉCTRICO CHILENO DIGA NO A LA LIBERALIZACIÓN DEL MERCADO SPOT ${ }^{1}$
}

\section{Soledad Arellano}

Este trabajo argumenta que la liberalización del mercado spot de la industria eléctrica chilena no es una alternativa de política recomendable, pues existe un amplio potencial para ejercer poder de mercado. En particular, Endesa distorsionaría la asignación intertemporal del agua, forzando así al alza el precio spot, especialmente en períodos en que la demanda que enfrenta es menos elástica. Se analizan además dos medidas de política que podrían servir para mitigar el problema de competencia: la venta de activos por parte de Endesa y la obligación de vender parte de la producción a través de contratos. Si bien ambas alternativas efectivamente lograrían reducir el problema de poder de mercado, ninguna de ellas constituye en la práctica una garantía de que el mencionado problema no se presentará. La primera alternativa tiene un problema de factibilidad práctica, mientras que el funcionamiento de la segunda no puede ser garantizado en el mediano y largo plazo.

M. Soledad Arellano. Ingeniero Comercial y Magíster en Economía de la Pontificia Universidad Católica de Chile. Doctor en Economía, Massachusetts Institute of Technology. Profesora investigadora del Centro de Economía Aplicada (CEA), Departamento de Ingeniería Industrial, Universidad de Chile. Email: sarellano@dii.uchile.cl

${ }^{1}$ Una versión previa de este trabajo se presentó en el Encuentro de la Sociedad de Economía de Chile 2004. Agradezco los comentarios de Alexander Galetovic, Ronald Fischer y los de un árbitro anónimo, así como también el financiamiento entregado por Fundación Andes (Proyecto C-13860). 


\section{Introducción}

\section{Cada cierto tiempo surgen voces que promueven la liberalización} del mercado spot eléctrico chileno. Este artículo muestra que tal idea no es buena, pues en caso de desregularse el mercado spot los generadores ejercerían poder de mercado, lo que resultaría en precios muy superiores al precio competitivo. La posición dominante es especialmente relevante en el caso de Endesa. Este artículo ilustra también los resultados de diversas políticas que deberían acompañar a la liberalización del mercado spot para mitigar el ejercicio de poder de mercado, destacando que éstas son o difíciles de implementar o inciertas en cuanto a su efectividad en el largo plazo. La recomendación de este trabajo es eliminar la posibilidad de liberalizar el mercado spot del conjunto de alternativas disponibles para reformar el sector eléctrico chileno.

Ya es casi una constante que en la discusión pública se esté analizando una o más propuestas para reformar el funcionamiento del sector eléctrico chileno. Estas propuestas, que se enmarcan en la ola de desregulación que se ha estado llevando a cabo en los últimos años a nivel mundial, usualmente apuntan a flexibilizar de uno u otro modo la operación del siste$\mathrm{ma}^{2}$. Sin lugar a dudas el cambio más radical que se ha planteado —en el año 2000 incluso circuló un proyecto de ley al respecto— es la liberalización del mercado spot, propuesta consistente en implementar un esquema de bolsa de energía, dejando que el precio se determine en base a las ofertas hechas por generadores y consumidores ${ }^{3}$.

La liberalización del mercado spot chileno conlleva al menos dos peligros: el ejercicio de poder de mercado y el abandono del despacho a costo marginal. La evidencia empírica indica que la primera práctica resulta en precios mayores que los competitivos y en una asignación de recursos ineficiente. El incentivo para ejercer poder de mercado es mayor en aquellos períodos en que la demanda que enfrentan los productores es menos elástica. En el caso de sistemas térmicos, los generadores maximizan sus utilida-

2 Algunos mercados en los que se ha desregulado la industria son Gran Bretaña, varios estados en Estados Unidos (PJM, California, New England Pool), Argentina, Colombia, Australia y Nueva Zelanda, entre otros.

${ }^{3}$ Es importante notar que el esquema de competencia que considera este trabajo no es exactamente igual al que contemplaba tal proyecto de ley. Éste consideraba que el despacho se haría en base a las ofertas de precio pero mantenía en forma transitoria la regulación de precio de nudo calculado en base a simulaciones de la operación del sistema con despacho según costo marginal. Luego en tales circunstancias el precio que paga el consumidor final no necesariamente coincide con el precio al cual se realizan las transacciones en el mercado spot. En este trabajo se asume que la liberalización es completa, por lo que el precio que paga el consumidor final es el que determina el mercado spot. 
des por la vía de restringir su producción (ver por ejemplo Borenstein y Bushnell, 1999; Borenstein et al., 2002, y Joskow y Kahn, 2002). En un sistema hidráulico (o mixto) los productores pueden ejercer poder de mercado no sólo por la vía de restringir la producción total sino también distorsionando la asignación intertemporal del agua de los embalses. Esta estrategia, no disponible para generadores con parque térmico, permite manipular los precios en forma sutil y poco observable directamente. Arellano (2004) muestra que en estos sistemas, el incentivo para ejercer poder de mercado está fuertemente determinado por la diferencia en la elasticidad precio de la demanda residual que se observa entre períodos. En particular, el productor tiene incentivo para subutilizar el agua disponible en períodos en que la demanda es menos elástica y para sobreutilizarla en períodos en que la demanda residual es más elástica, comparado con el comportamiento que tendría un productor competitivo.

Hay dos elementos estructurales del sistema chileno que determinan que el problema de poder de mercado sea una amenaza real: el alto grado de concentración de la industria en el segmento generación y la predominancia de los recursos hidráulicos asociados a embalses.

La evidencia empírica indica que el objetivo de establecer un mercado competitivo requiere contar con un número adecuado de empresas generadoras que compitan en el mercado mayorista. La experiencia inglesa, exitosa en numerosos aspectos, es un buen ejemplo de los problemas que un alto grado de concentración puede ocasionar, especialmente en relación con las prácticas anticompetitivas ${ }^{4}$. Este riesgo es especialmente relevante en el caso de la industria chilena, dado el alto grado de concentración a nivel de generación. En efecto, si bien existen alrededor de 20 empresas generadoras en el SIC, tres grupos económicos - Endesa, Gener y Colbún- controlan directa e indirectamente alrededor del 90\% de la capacidad instalada (diciembre 2004). Por otro lado, el 60\% de la capacidad instalada corresponde a plantas hidráulicas, de la cual $72 \%$ son plantas asociadas a embalses con diversa capacidad de regulación. Endesa no sólo es en términos absolutos la empresa más grande del sistema, sino que además posee el $68 \%$ de los recursos hidráulicos, proporción que aumenta a $81 \%$ si sólo se consideran los embalses.

La liberalización del mercado spot no sólo afecta al precio en el cual se transaría la energía en el mercado spot, sino que también afecta a la operación misma del sistema. En particular, un cambio de este tipo obligaría a abandonar el despacho del parque generador en base al costo marginal de

${ }^{4}$ Para más detalles de la experiencia desregulatoria en Estados Unidos y Gran Bretaña, ver Joskow (2002). 
las centrales para dar paso al despacho en base a los precios ofertados por los generadores. En efecto, aun cuando el despacho se siga realizando en base a orden de mérito, en un esquema de bolsa de energía el despacho no utiliza la información de costo marginal de las plantas sino las ofertas entregadas por los productores. Si estas ofertas no son competitivas, en la práctica el despacho no se realizará en base al costo marginal, como se hace actualmente, dejando de lado uno de los pilares que garantizan la operación eficiente del sistema. Las características del sistema chileno a nivel mayorista y en particular el mecanismo de operación del mercado spot limitan las posibilidades de declarar costos marginales superiores a los efectivos, atenuando el problema de poder de mercado y garantizando que las plantas en operación son efectivamente las de menor costo.

Este trabajo analiza cualitativa y cuantitativamente el efecto de la liberalización del mercado spot en el Sistema Interconectado Central, SIC. En particular, muestra que esta alternativa resultaría en un amplio ejercicio de poder de mercado, especialmente por parte de Endesa. En tales circunstancias esta empresa no sólo tiene incentivos para reducir la producción de su parque térmico sino que también para distorsionar la asignación intertemporal del agua de sus embalses entre períodos. La sociedad como un todo incurre en un costo de bienestar no sólo porque la producción total es menor que la de equilibrio, sino además porque las plantas utilizadas en la producción no corresponden a aquellas que minimizan el costo total de operación. Los resultados indican además que Gener, a pesar de contar con un espacio limitado para aumentar los precios, es, en términos relativos, el gran beneficiado por esta medida. Posteriormente se analiza el efecto de dos medidas que la autoridad podría implementar para mitigar el problema de competencia en este mercado: i) la venta de activos por parte de Endesa, y ii) obligar a los generadores a vender a través de contratos una proporción de su demanda. Si bien ambas medidas son teóricamente exitosas en cuanto a acercar el equilibrio de mercado al equilibrio competitivo, no está claro cuán factibles serán realmente en la práctica. En efecto, la factibilidad de implementar la primera medida es cuestionable, especialmente cuando se toman en consideración los costos políticos involucrados. En el caso de la alternativa de los contratos, las condiciones de la industria no permiten asegurar su factibilidad en el mediano plazo.

En consecuencia, los resultados de este trabajo recomiendan descartar la alternativa de liberalizar el mercado spot eléctrico en Chile. La implementación de esta reforma se traducirá en un problema de competencia muy difícil de limitar a través de medidas mitigadoras, ya sea por motivos de orden político-práctico, como de factibilidad económica en el mediano plazo. 
Este trabajo se organiza de la siguiente manera. En la próxima sección se describen brevemente las características del segmento generación de la industria en Chile. En la sección 3 se describe el equilibrio que resultaría en caso de liberalizar el mercado spot y se lo compara con el equilibrio competitivo. En la sección 4 se presentan las posibles medidas que se podrían implementar para mitigar el problema de poder de mercado y sus resultados. La última sección presenta las conclusiones de este trabajo.

\section{Funcionamiento del segmento generación de la industria eléctrica en Chile}

La electricidad en Chile se provee a través de cuatro sistemas eléctricos no interconectados entre sí, siendo el sistema interconectado central (SIC) el de mayor tamaño. Su parque generador combina centrales térmicas con centrales hidráulicas, incluyendo tanto centrales de pasada como centrales asociadas a algún embalse con capacidad de regulación. La demanda máxima del sistema en el año 2002 ascendió a 4.800 MW y la generación bruta a casi 32.000 GWh, 70\% de la cual corresponde a generación hidráulica. Como se observa en la Tabla $\mathrm{N}^{\circ} 1$, la propiedad del parque generador de este sistema está concentrada principalmente en tres grupos económicos asociados a Endesa, Gener y Colbún, que en conjunto controlan un 92\% de la capacidad instalada total del sistema ${ }^{5}$. Estas empresas difieren tanto en tamaño como en composición de su parque generador, pues mientras el portfolio de Endesa y Colbún incluye plantas térmicas e hidráulicas con capacidad de regulación, el portfolio de Gener es principalmente térmico.

En el sistema chileno, los generadores pueden comercializar su energía a través de tres mecanismos. En primer lugar, pueden vender a los denominados consumidores libres, los que no están sujetos a regulación de precio. Adicionalmente pueden vender parte de su producción a las empresas distribuidoras a través de contratos, siendo el precio contratado el precio de nudo ${ }^{6}$. Finalmente, los productores también están autorizados a

${ }^{5}$ En el texto se presentan los datos para diciembre de 2002, que es el período que se usó en las simulaciones reportadas en este artículo. Las participaciones relativas en diciembre de 2004 no variaron mayormente: las tres mayores empresas controlan el $90 \%$ de la capacidad instalada del sistema. El 60\% del total corresponde a plantas hidráulicas y un $72 \%$ de esa capacidad está asociada a embalses con capacidad de regulación. Endesa controla el $68 \%$ del total de los recursos hidráulicos.

${ }^{6}$ De acuerdo con la última reforma a la ley eléctrica, el precio fijado en el contrato se mantendrá constante por un período determinado en las bases de la licitación, con un tope de 15 años. 


\begin{tabular}{lrrrrr}
\hline Grupo económico & Térmica & $\begin{array}{c}\text { Hidráulica- } \\
\text { pasada }\end{array}$ & $\begin{array}{c}\text { Hidráulica- } \\
\text { embalse }\end{array}$ & Hidráulica & Total \\
\hline & & & & & \\
Endesa ("E") & 839 & 429 & 2.263 & 2.693 & 3.532 \\
Gener ("G") & 1.237 & 245 & 0 & 245 & 1.482 \\
Otras ("F") & 606 & 628 & 490 & 1.118 & 1.723 \\
Total & 2.682 & 1.302 & 2.753 & 4.055 & 6.737 \\
\hline
\end{tabular}

Fuente: CDEC-SIC.

realizar transacciones de energía y potencia entre ellos en el mercado spot, las que son valorizadas a costo marginal de corto plazo.

El Centro de Despacho Económico de Carga (CDEC) es el organismo encargado de la labor de despacho, es decir, de decidir qué unidades del sistema deben producir y cuánto. El despacho se realiza según el criterio de “orden de mérito”, en virtud del cual las unidades son llamadas a operar en base a su costo declarado, siendo las más baratas las llamadas a producir primero hasta que la producción sea suficiente para satisfacer la demanda en cada momento del tiempo. El despacho es, en consecuencia, totalmente independiente de los contratos comerciales que los productores puedan haber firmado anteriormente. La coexistencia de despacho obligatorio según orden de mérito y de contratos comerciales determina que, la mayor parte del tiempo, los volúmenes de energía contratados no coincidan con lo producido, lo que da origen a generadores con posiciones deficitarias y con posiciones de superávit. Para hacer frente a los compromisos comerciales previamente adquiridos, los generadores deficitarios deben comprar a los que tienen superávit sus excedentes de producción en el denominado mercado spot. El precio al que se transa la energía en este mercado — el costo marginal de corto plazo - es calculado por el CDEC para cada hora y corresponde al costo de operación de la central cuya producción permite igualar la oferta con la demanda.

El riesgo de sobrestimar los costos de producción como una forma de impulsar los precios sobre el nivel competitivo en el mercado spot chileno es limitado, debido a ciertos aspectos de la regulación. El primero es la desvinculación entre el precio de nudo — es decir el precio que los generadores reciben por la venta de su producción a los clientes regulados (e indirectamente a sus clientes libres) - y el costo marginal de corto plazo efectivo. Éste es a su vez el precio que deben pagar en el mercado spot los generadores en posición deficitaria por la energía necesaria para suplir la 
diferencia entre la energía comprometida en los contratos y la producida (según órdenes del despacho). En consecuencia, el generador deficitario tiene un fuerte incentivo para vigilar que quien vende declare sus verdaderos costos. La alternancia en las posiciones deficitarias y con superávit de los generadores a través del tiempo refuerza la vigilancia mutua, forzando a que los costos declarados coincidan con los costos marginales verdaderos. Esta misma característica limita la posibilidad de colusión entre los agentes. En consecuencia, es precisamente el mecanismo de operación del mercado spot el que limita las posibilidades de ejercer poder de mercado ya sea en forma individual o conjunta ${ }^{7}$.

\section{Equilibrio de mercado bajo liberalización del mercado spot}

Para analizar el efecto de la liberalización del mercado spot se simulará el equilibrio de mercado utilizando como base el modelo propuesto por Arellano (2003) y la información de demanda y costos del Sistema Interconectado Central correspondiente al año 2002.

La existencia de un parque hidráulico determina que al decidir cuánto producir en cada período de tiempo, el productor debe considerar el hecho de que utilizar más agua hoy significa que menos queda disponible para mañana. Para tomar en cuenta la dependencia intertemporal se asume que los productores maximizan sus utilidades en un horizonte de planificación de un mes, el cual puede ser representado a través de seis subperíodos de una hora de duración cada uno, a los que se les designa como $t$. Estos subperíodos están ordenados de mayor a menor, de modo que $t=1$ indica el período de mayor demanda, y $t=6$, el período de menor demanda de mercado.

El modelo incorpora el hecho de que no todos los recursos hidráulicos son iguales. Sólo aquellas centrales con un embalse aguas arriba tienen efectivamente la posibilidad de acumular agua entre períodos, y en consecuencia pueden decidir cuándo acumular o liberar el agua para generar energía eléctrica. La imposibilidad de hacer uso estratégico de las centrales de pasada se incorpora en el modelo por la vía de modelar la producción de tales plantas como generación obligada (no estratégica). A partir de este

${ }^{7}$ Se debe tener presente, sin embargo, que si bien es cierto que en un mercado como el chileno es difícil ejercer poder de mercado a través de estrategias de precio, los generadores pueden influir indirectamente en ellos a través de su decisión de inversión. Esta decisión puede afectar a los costos marginales a través del tamaño y de la composición de su parque generador. Luego, el mercado spot es de todos modos susceptible de ser víctima de una forma más sutil de ejercicio de poder de mercado. Para más detalles, ver Arellano y Serra (2005). 
momento toda mención a generación hidráulica hará referencia a la producción originada en los embalses, a menos que se mencione explícitamente lo contrario.

Se supone que la industria está integrada por dos tipos de empresas: un conjunto de pequeñas empresas, que se comportan como tomadores de precio, y dos empresas de mayor tamaño que compiten a la Cournot y que fueron construidas en base a las características de Gener y Endesa ${ }^{8}$. La coexistencia de ambos tipos de empresas determina que Endesa y Gener no enfrenten toda la demanda de mercado, sino la demanda residual, esto es, la demanda de mercado neta de la oferta de los pequeños productores.

Gener y Endesa difieren entre sí en cuanto al tamaño y a la composición de su parque generador. Mientras la primera controla sólo plantas térmicas, la segunda posee también plantas hidráulicas asociadas a embalses con capacidad de regulación. Esto determina que la única decisión estratégica que Gener puede tomar sea cuánto producir en cada momento del tiempo. Endesa, en cambio, puede decidir cuánto producir con cada una de sus plantas térmicas e hidráulicas y, asimismo, cuándo utilizar el agua disponible.

El modelo teórico y los datos utilizados en la simulación se discuten con mayor detalle en el Apéndice de este estudio. Como todo modelo, el que se usa en este estudio es una representación simplificada de la realidad. En particular, en este caso no se consideraron aspectos de competencia dinámica ni las características del sistema de transmisión, por lo que los resultados podrían subestimar el problema de poder de mercado. Un elemento adicional para tener en cuenta al momento de analizar los resultados es que a diferencia del esquema planteado en el proyecto de ley del año 2000, el modelo contempla un completo traspaso del precio spot al consumidor final (al menos para el período de transición). Lo anterior determina que este modelo subestime el poder de mercado que podría ejercerse en el contexto de tal proyecto por dos motivos. En primer lugar, dado que el consumidor percibe directamente el precio spot, el modelo permite que éste reaccione al ejercicio de poder de mercado por la vía de reducir su consumo. Por otro lado, cuando el precio en el mercado spot no está relacionado con el precio que paga el consumidor final, el "costo" de ejercer poder de mercado, especialmente para un generador que no está muy contratado, es relativamente bajo.

${ }^{8}$ Se escogió la opción de duopolio, pues en todas las estimaciones del equilibrio que se realizaron para el caso de un triopolio, el potencialmente tercer productor con poder de mercado — Colbún — se comporta como tomador de precios. 
Para efectos de la simulación del equilibrio de mercado resultante se consideraron dos escenarios de competencia: i) ningún productor tiene poder de mercado, y ii) Endesa y Gener tienen poder de mercado y se comportan à la Cournot. Los resultados de cada escenario se presentan en las Tablas $\mathrm{N}^{\mathrm{o}} 2$ bajo la denominación "Equilibrio competitivo" y "Equilibrio Cournot”, respectivamente.

TABLA N ${ }^{\circ} 2: \quad$ RESULTADOS SIMULACIÓN MODELO BASE $(\varepsilon=-1 / 3)$

Tabla 2a: Equilibrio competitivo

\begin{tabular}{lccccccc}
$\mathrm{t}$ & $T_{E}$ & $H_{E}$ & $T_{G}$ & $T_{F}$ & $H_{F}$ & $\mathrm{Q}$ & $\begin{array}{c}\text { Precio } \\
{[8]}\end{array}$ \\
& {$[2]$} & {$[3]$} & {$[4]$} & {$[5]$} & {$[6]$} & {$[7]$} & \\
\hline & & & & & & & \\
1 & 680,3 & $1.908,3$ & 950,6 & 556,7 & 417,3 & $4.907,1$ & 33,2 \\
2 & 680,3 & $1.747,7$ & 950,6 & 556,7 & 188,3 & $4.517,5$ & 33,2 \\
3 & 680,3 & $1.617,1$ & 950,6 & 556,7 & 146,5 & $4.345,2$ & 33,2 \\
4 & 680,3 & $1.219,6$ & 950,6 & 556,7 & 146,5 & $3.947,7$ & 33,2 \\
5 & 680,3 & 833,9 & 950,6 & 556,7 & 146,5 & $3.562,0$ & 32,7 \\
6 & 680,3 & 796,2 & 950,6 & 556,7 & 146,5 & $3.524,3$ & 27,9 \\
& & & & & & & \\
\hline
\end{tabular}

Tabla 2b: Equilibrio Cournot

\begin{tabular}{lrcccccc}
$\mathrm{t}$ & \multicolumn{1}{c}{$T_{E}$} & $H_{E}$ & $T_{G}$ & $T_{F}$ & $\begin{array}{c}H_{F} \\
{[6]}\end{array}$ & $\begin{array}{c}\mathrm{Q} \\
{[7]}\end{array}$ & $\begin{array}{c}\text { Precio } \\
{[8]}\end{array}$ \\
\hline & {$[2]$} & {$[3]$} & {$[4]$} & {$[5]$} & {$[6]$} & & \\
\hline 1 & 121,5 & $1.622,5$ & 950,6 & 556,7 & 417,3 & $4.062,7$ & 51,4 \\
2 & 101,7 & $1.562,0$ & 950,6 & 556,7 & 188,3 & $3.753,3$ & 49,7 \\
3 & 96,7 & $1.501,8$ & 950,6 & 556,7 & 146,5 & $3.646,3$ & 48,3 \\
4 & 107,2 & $1.292,6$ & 950,6 & 556,7 & 146,5 & $3.447,6$ & 44,0 \\
5 & 79,1 & $1.136,3$ & 909,7 & 556,7 & 146,5 & $3.222,3$ & 40,0 \\
6 & 111,4 & $1.008,6$ & 839,1 & 556,7 & 146,5 & $3.056,3$ & 38,0 \\
\hline
\end{tabular}

Donde $\quad \mathrm{T}_{\mathrm{i}}=$ producción térmica de la empresa "i” en la hora $t(\mathrm{MW})$;

$\mathrm{H}_{\mathrm{i}}=$ producción hidráulica de la empresa "i" en la hora $t(\mathrm{MW})$;

$\mathrm{i}$ = E (Endesa), G (Gener), F (pequeños productores);

$\mathrm{Q}=$ producción total en la hora $t(\mathrm{MW})$.

Resultado 1: La liberalización del mercado spot genera condiciones favorables para el ejercicio de poder de mercado. Éste no sólo se traduce en menor producción y precios más altos que los competitivos, sino además en una asignación intertemporal del agua que es ineficiente. En particular, en los períodos de mayor demanda se usa relativamente 
poca agua, mientras que en los períodos de baja demanda se utiliza relativamente mucha agua.

Según se observa en las Tablas $\mathrm{N}^{\circ} 2$ (columnas [7] y [8]), en el equilibrio Cournot el nivel de producción es menor y los precios son mayores que en el equilibrio competitivo. Esta diferencia es mayor en los períodos de mayor demanda. Obsérvese que es principalmente Endesa la que restringe su producción, pues en los períodos de punta Gener se comporta como tomador de precios, produciendo al máximo de su capacidad. En otras palabras, es Endesa la que efectivamente ejerce poder de mercado, pues sus decisiones de producción determinan que los precios sean tan altos, que la estrategia óptima para Gener es producir al máximo de su capacidad.

La Figura $\mathrm{N}^{\circ} 1$ muestra claramente que la distorsión con respecto al equilibrio competitivo es mayor en los períodos de mayor demanda, los que, como consecuencia de las restricciones de capacidad de los productores rivales, coinciden con los períodos en que la demanda residual que enfrenta Endesa es menos elástica. Como resultado de lo anterior, el margen que esta empresa aplica, calculado según el índice de Lerner, alcanza un máximo de $73 \%$ en el período de mayor demanda, y un mínimo de $64 \%$ en el período de menor demanda, obteniendo en promedio un $70 \%$.

Endesa ejerce poder de mercado restringiendo la producción de sus plantas térmicas y distorsionando la asignación intertemporal del agua. Según se observa claramente en la Figura $\mathrm{N}^{\circ}$ 2, Endesa asigna relativamente poca agua a los períodos de mayor demanda y relativamente mucha a los períodos de baja demanda. Como resultado, la diferencia de carga neta entre los períodos de punta y no punta se reduce cuando el agua se asigna en forma competitiva, mientras que aumenta cuando es un productor con po-

FIGURA N ${ }^{\circ}$ 1: $\quad$ PRECIO DE EQUILIBRIO EN COMPETENCIA VS COURNOT

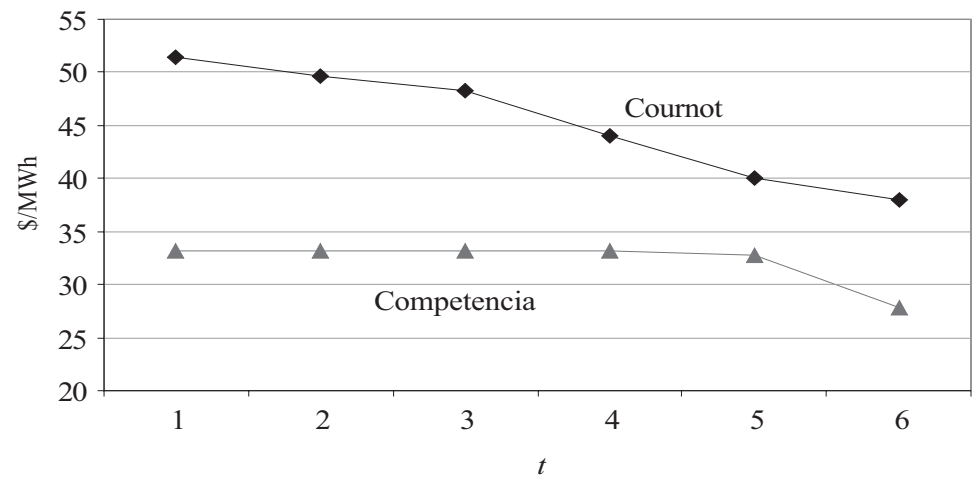


FIGURA No 2: $\quad$ ASIGNACIÓN INTERTEMPORAL DEL AGUA EN COMPETENCIA VS COURNOT

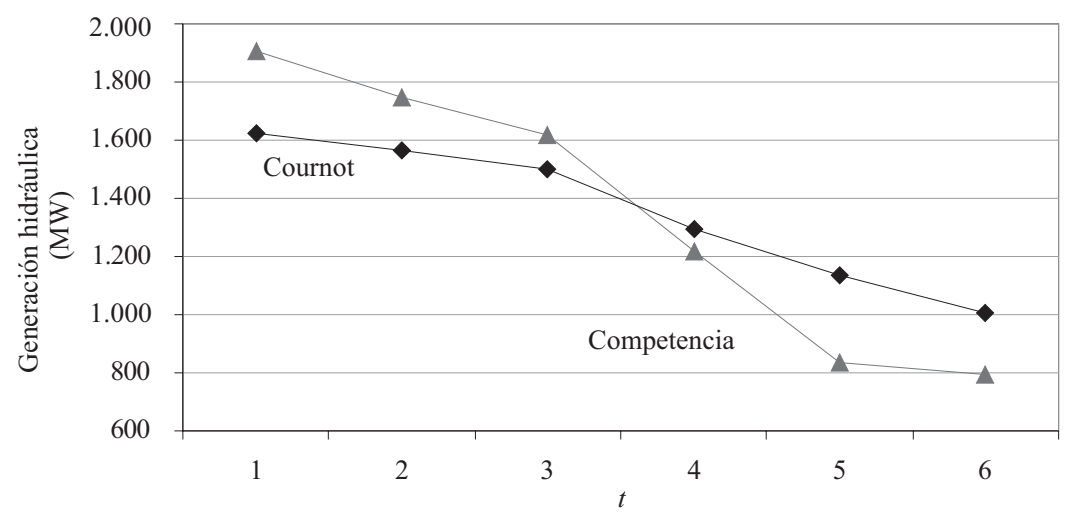

der de mercado quien toma tal decisión. Estos resultados están en línea con lo encontrado previamente en la literatura, pues, tal como se afirmó, los primeros coinciden con períodos en que la demanda residual es menos elástica.

La fuente de poder de mercado de Endesa es su gran capacidad de generación asociada a los embalses. De hecho, en años secos esta empresa pierde toda su capacidad para influir en el precio de mercado, y en tales circunstancias es Gener quien está en posición de hacerlo ${ }^{9}$. Obsérvese que Gener, a pesar de concentrar un 22\% del parque generador, sólo tiene incentivo para restringir la producción -y de este modo para ejercer poder de mercado- en condiciones de baja demanda. Detrás de este resultado está el hecho de que una parte importante de la capacidad térmica de esta empresa comprende plantas de base.

Un ejercicio interesante consiste en constatar cuánto se beneficia o perjudica cada uno de los agentes como resultado del ejercicio de poder de mercado. Si bien todos los productores se benefician —el excedente total de los productores aumenta en 37\%-, Gener y los pequeños productores son quienes más se benefician en términos relativos (72\% y 60\%, respectivamente, comparado con un $19 \%$ en el caso de Endesa) ${ }^{10}$. La razón de este resultado es clara: tanto en el equilibrio Cournot como en el equilibrio competitivo, estos generadores producen prácticamente lo mismo, pero en el primer caso el precio al cual se transa la energía en el mercado es ostensible-

${ }^{9}$ Para más detalles, ver Arellano (2003).

${ }^{10}$ En términos absolutos, Endesa es la empresa más grande de la industria y la que obtiene las mayores utilidades (53\% del total). 
mente mayor. Como era de esperar, el excedente de los consumidores se ve reducido debido al poder de mercado; en particular éste cae alrededor de 27\%. En términos agregados, el bienestar social disminuye en 5,1\%

Resultado 2: El ejercicio de poder de mercado resulta en un equilibrio que es ineficiente tanto a nivel asignativo como productivo, por lo que el bienestar social es menor que el que se obtendría en un equilibrio competitivo.

Este resultado de menor bienestar no sólo está asociado a que la producción total es menor que la socialmente óptima y a que el precio es mayor. Un costo adicional resulta del hecho de que el despacho de las plantas no se realiza en base a sus respectivos costos marginales. En consecuencia, las plantas que operan no son las que minimizan el costo de operación. Esto es así porque Endesa restringe la producción de sus plantas térmicas, incluyendo aquellas con bajo costo de operación, y paralelamente los pequeños productores producen al máximo de su capacidad, con plantas cuyo costo de operación es mucho mayor que el costo de operación de las plantas que Endesa sacó del mercado. El abandono del despacho a costo marginal determina que los costos totales sean $20 \%$ superiores a aquellos en que se incurrirían si las plantas más eficientes del sistema produjeran el mismo volumen de energía térmica que resulta en el equilibrio Cournot. Lo anterior determina que un $27 \%$ del menor bienestar que resulta en el equilibrio Cournot se explique exclusivamente por la no minimización de los costos.

\section{Medidas mitigadoras}

Los resultados presentados en la sección anterior clarifican lo riesgoso y costoso que puede ser para la sociedad la liberalización del mercado spot. En consecuencia, si la autoridad económica está aún dispuesta a seguir adelante con esta reforma, es imprescindible que implemente medidas adicionales tendientes a mitigar el potencial problema de competencia. A continuación se analiza cualitativa y cuantitativamente el efecto de dos posibles alternativas para aplicar: i) la enajenación de parte del parque generador de Endesa, y ii) obligar a los generadores a vender un porcentaje de su producción a través de contratos.

Es importante destacar que ambas medidas ya han sido utilizadas en el extranjero con el mismo objetivo. Así por ejemplo, cuando Australia y

${ }^{11}$ Se debe tener en cuenta que la magnitud del cambio en el bienestar social está asociada al supuesto de elasticidad de demanda utilizado. 
Argentina desregularon la industria eléctrica, las empresas originales fueron divididas en varias empresas de menor tamaño que posteriormente fueron - en parte - privatizadas. Por otro lado, los generadores en Australia fueron obligados a vender su producción a comercializadores a través de contratos, de modo que éstos pudieran respaldar sus ventas al consumidor final. En este caso los precios de los contratos fueron fijados por el gobierno en niveles considerablemente altos. En el caso del Reino Unido, si bien al momento de ser privatizadas las empresas generadoras tenían una alta participación de mercado, éstas también tenían un alto porcentaje de su producción vendida a través de contratos ( $87 \%$ en el caso de National Power y $88 \%$ en el caso de Power Gen, porcentajes que se redujeron a $72 \%$ y $70 \%$, respectivamente, después de que expiró el primer conjunto de contratos a un año). Adicionalmente, y en respuesta a la amenaza que el regulador hizo a estas empresas de acusarlas ante la Monopolies and Mergers Commission, los productores no sólo aceptaron someterse a un price cap sino que también aceptaron vender una parte de sus plantas térmicas a terceros productores ${ }^{12}$.

\subsection{Venta de activos}

A continuación se analiza el equilibrio que resultaría si Endesa se viera obligada a vender parte de su parque generador. Se analiza en forma separada la venta del parque térmico y la de las plantas hidráulicas asociadas a embalses.

\subsubsection{Venta del parque térmico}

La organización industrial que resulte una vez concluida la enajenación depende de la manera como ésta se efectúe y de quiénes sean los compradores. En particular, podrían producirse dos casos extremos. El peor, en términos de potencial para comportamientos anticompetitivos, es la venta de todo el parque a un único productor, dando origen a un triopolio. El mejor caso es la venta de las plantas en forma separada a diversos productores tomadores de precio en forma individual. En este trabajo se tomó la opción de mostrar lo mínimo que se podría ganar en caso de implementar esta medida, por lo que sólo se reportarán los resultados para el caso en que todo el portfolio térmico de Endesa se vende a un tercer productor

${ }^{12}$ Para más detalles, ver Wolak (1999) y ENRE (1995, 1997) 


\begin{tabular}{ccccccccc}
\hline $\mathrm{t}$ & $\begin{array}{c}T_{E} \\
{[2]}\end{array}$ & $\begin{array}{c}H_{E} \\
{[3]}\end{array}$ & $\begin{array}{c}T_{G} \\
{[4]}\end{array}$ & $\begin{array}{c}T_{N E} \\
{[5]}\end{array}$ & $\begin{array}{c}T_{F} \\
{[6]}\end{array}$ & $\begin{array}{c}H_{F} \\
{[7]}\end{array}$ & $\begin{array}{c}\mathrm{Q} \\
{[8]}\end{array}$ & $\begin{array}{c}\text { Precio } \\
{[9]}\end{array}$ \\
\hline & & & & & & & \\
\hline 1 & 0,0 & $1.568,1$ & 942,3 & 680,3 & 556,7 & 417,3 & $4.558,7$ & 40,7 \\
2 & 0,0 & $1.514,6$ & 888,8 & 680,3 & 556,7 & 188,3 & $4.222,6$ & 39,6 \\
3 & 0,0 & $1.471,0$ & 845,3 & 680,3 & 556,7 & 146,5 & $4.093,8$ & 38,6 \\
4 & 0,0 & $1.315,4$ & 759,1 & 680,3 & 556,7 & 146,5 & $3.852,0$ & 35,3 \\
5 & 0,0 & $1.173,6$ & 633,2 & 680,3 & 556,7 & 146,5 & $3.584,2$ & 32,2 \\
6 & 0,0 & $1.081,1$ & 633,2 & 603,6 & 556,7 & 146,5 & $3.415,1$ & 30,2 \\
\hline
\end{tabular}

Donde $\mathrm{T}_{\mathrm{i}}=$ producción térmica de la empresa "i" en la hora $t(\mathrm{MW})$;

$\mathrm{H}_{\mathrm{i}}=$ producción hidráulica de la empresa "i”" en la hora $t(\mathrm{MW})$;

$\mathrm{i}=\mathrm{E}$ (Endesa), $\mathrm{G}$ (Gener), $\mathrm{F}$ (pequeños productores), NE;

$\mathrm{Q}=$ producción total en la hora $t(\mathrm{MW})$.

"NE", dando origen a un triopolio ${ }^{13}$. En tales circunstancias, la industria estaría integrada por tres productores: Endesa, con un parque puramente hidráulico, y Gener y “NE” con un portfolio puramente térmico. La única herramienta con que contaría Endesa para ejercer poder de mercado sería el uso de las plantas hidráulicas, en particular la asignación intertemporal del agua. Los resultados de la simulación se presentan en la Tabla $\mathrm{N}^{\circ} 3$.

El efecto más notorio de la enajenación de las plantas térmicas de Endesa es el aumento en la cantidad total producida y la consecuente reducción en el precio, siendo el cambio proporcionalmente mayor en los períodos de mayor demanda. A primera vista esto podría ser un indicador de que esta medida consiguió eliminar el poder de mercado que ejercía anteriormente Endesa. Sin embargo, al analizar la producción a nivel de cada empresa, es claro que no es así. En efecto, el aumento en la producción total proviene principalmente de la mayor producción de las plantas térmicas que originalmente estaban en manos de Endesa. De hecho, Gener ve su posición en el mercado relativamente fortalecida, por eso restringe levemente su producción con respecto al equilibrio en duopolio, y, como se puede observar claramente en la Figura $N^{0} 3$, Endesa prácticamente no altera su estrategia en torno a cómo asignar el agua en forma intertemporal. Esto indica que esta medida no logra eliminar la capacidad de Endesa de influir en el precio de mercado. Endesa sigue controlando la herramienta que constituye la base de su fuerte posición en el mercado: las plantas hidráulicas. El prin-

13 En Arellano (2003) se presentan los resultados para el caso en que el parque generador de Endesa se venda a productores tomadores de precios (datos del año 2000). 
FIGURA N N $^{\circ}$ EFECTO VENTA DE ACTIVOS EN ASIGNACIÓN INTERTEMPORAL DEL AGUA

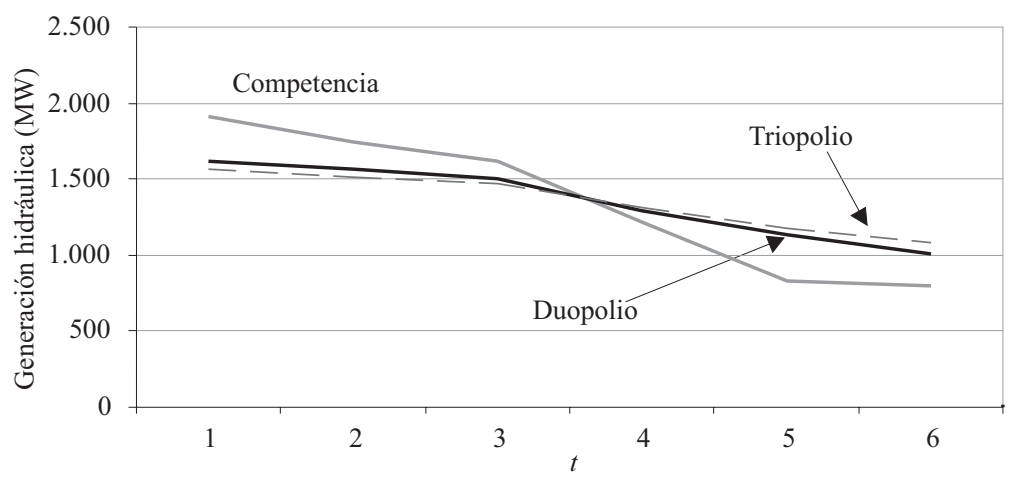

cipal beneficio de esta medida resulta del mejor uso de la capacidad instalada, pues las plantas térmicas enajenadas producen en niveles cercanos a los eficientes ${ }^{14}$.

\subsubsection{Venta del parque hidráulico}

Los resultados anteriores muestran que la posición de Endesa en la industria está fuertemente determinada por su capacidad para manejar los embalses. Luego, una segunda medida a la que se podría recurrir para mitigar el problema de competencia sería obligar a Endesa a vender la totalidad o parte de su parque generador hidráulico, de modo que éste sea despachado con un criterio competitivo. La implementación de esta medida no necesariamente requiere de la enajenación de las plantas; bastaría con traspasar el manejo de los embalses a una entidad independiente, como podría ser el CDEC. De llevarse a cabo tal medida, la industria estaría conformada por dos grandes generadores, Endesa y Gener, ambos con un portfolio de generación completamente térmico.

A diferencia del caso base, todo el parque hidráulico, y no sólo el que inicialmente estaba en manos de los pequeños productores, se despacha con el criterio de eliminar (o reducir) los peaks y de este modo igualar el precio entre períodos. La Figura $\mathrm{N}^{\mathrm{o}} 4$ muestra que, dado el volumen de agua involucrado (y de la energía equivalente), este criterio de asignación es efectivo en cuanto a este objetivo, pues la curva de carga neta queda prácti-

${ }^{14}$ El hecho de que el tercer productor utilice sus plantas en niveles prácticamente eficientes y no ejerza poder de mercado sugiere que el número de compradores del parque térmico de Endesa no es relevante. 
FIGURA N ${ }^{\circ}$ 4: CURVA DE CARGA Y ASIGNACIÓN INTERTEMPORAL EFICIENTE DEL AGUA

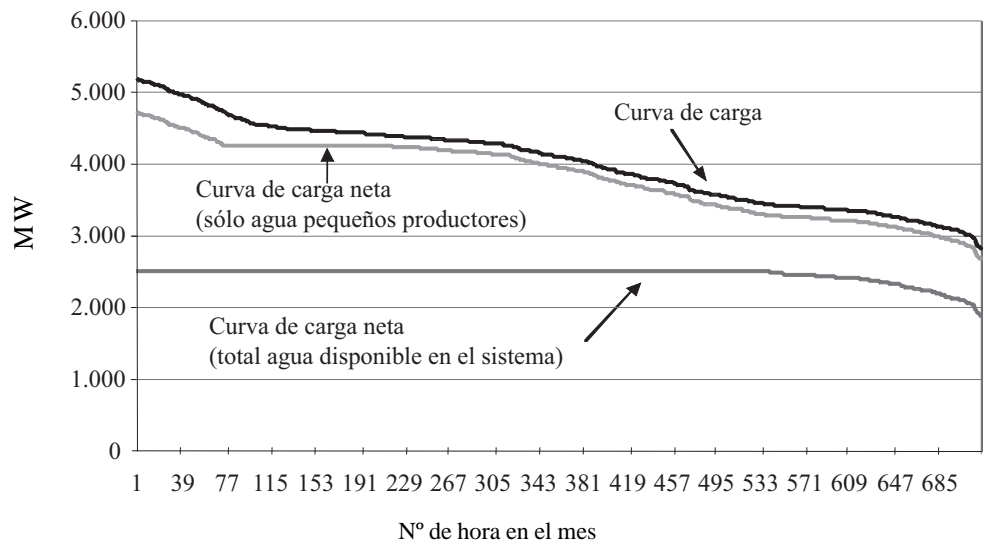

camente plana en la mayor parte del período. Esto a su vez determina que la demanda residual que los productores Cournot enfrentan en cada período no sólo sea relativamente más elástica que en el caso base, sino que además la diferencia entre períodos sea considerablemente menor.

Los resultados de la simulación se presentan en la Tabla $\mathrm{N}^{\circ} 4$. Al igual que en el caso anterior, el nivel de precios y el volumen de producción total se acercan a los de equilibrio. El comportamiento de los productores que explica este resultado, sin embargo, es distinto. En efecto, mientras la producción de Endesa coincide con la de un productor tomador de precios, Gener restringe su producción, compensando en parte la mayor producción térmica de Endesa. Lo anterior implica que la enajenación del parque hidráulico resulta en una completa pérdida de poder de mercado por parte de Endesa y en un fortalecimiento en la posición relativa de Gener en la industria.

Es interesante notar que el mercado alcanzaría un equilibrio similar en el caso de que Endesa enajenara sólo las centrales asociadas al embalse Laja ${ }^{15}$. Lo anterior sugiere que el eventual poder de mercado de esta empresa radica principalmente en el control del Laja.

Estos resultados indican que tanto la venta del parque térmico como la venta del parque hidráulico de Endesa permiten acercar el equilibrio de mercado al equilibrio competitivo. La segunda medida es más efectiva que la primera, pues apunta directamente al elemento en el que se basa la fortaleza de la posición que Endesa ostenta en la industria eléctrica. Aun en este

\footnotetext{
${ }^{15}$ Resultados no reportados. Para más detalles, ver Arellano (2003).
} 
TABLA No 4: EQUILIBRIO DE MERCADO POSVENTA DEL PARQUE HIDRÁULICO DE ENDESA

\begin{tabular}{ccccccccc}
\hline $\mathrm{t}$ & $\begin{array}{c}T_{E} \\
{[2]}\end{array}$ & $\begin{array}{c}H_{E} \\
{[3]}\end{array}$ & $\begin{array}{c}T_{G} \\
{[4]}\end{array}$ & $\begin{array}{c}H_{N E} \\
{[5]}\end{array}$ & $\begin{array}{c}T_{F} \\
{[6]}\end{array}$ & $\begin{array}{c}H_{F} \\
{[7]}\end{array}$ & $\begin{array}{c}\mathrm{Q} \\
{[8]}\end{array}$ & $\begin{array}{c}\text { Precio } \\
{[9]}\end{array}$ \\
\hline & & & & & & & & \\
1 & 680,3 & 0,0 & 807,0 & $1.908,3$ & 556,7 & 417,3 & $4.763,5$ & 36,3 \\
2 & 680,3 & 0,0 & 807,0 & $1.747,7$ & 556,7 & 188,3 & $4.373,9$ & 36,3 \\
3 & 680,3 & 0,0 & 807,0 & $1.617,1$ & 556,7 & 146,5 & $4.201,6$ & 36,3 \\
4 & 680,3 & 0,0 & 807,0 & $1.219,6$ & 556,7 & 146,5 & $3.804,1$ & 36,3 \\
5 & 680,3 & 0,0 & 795,1 & 833,9 & 556,7 & 146,5 & $3.406,4$ & 36,3 \\
6 & 680,3 & 0,0 & 683,2 & 796,2 & 556,7 & 146,5 & $3.256,8$ & 36,3 \\
\hline
\end{tabular}

Donde $\mathrm{T}_{\mathrm{i}}=$ producción térmica de la empresa "i" en la hora $t$ (MW);

$\mathrm{H}_{\mathrm{i}}=$ producción hidráulica de la empresa "i” en la hora $t(\mathrm{MW})$;

$\mathrm{i}=\mathrm{E}$ (Endesa), $\mathrm{G}$ (Gener), $\mathrm{F}$ (pequeños productores), NE;

$\mathrm{Q}=$ producción total en la hora $t(\mathrm{MW})$.

caso, sin embargo, persiste el problema de poder de mercado, pues en forma paralela al debilitamiento de la posición de Endesa, la posición de Gener se va fortaleciendo, pero sin alcanzar en ningún caso el poder que inicialmente ostenta la primera.

\subsection{Contratos}

Tanto la teoría económica como la evidencia empírica han mostrado que mientras mayor es el porcentaje de la producción que los generadores venden a través de contratos, menor es el incentivo para ejercer poder de mercado y, en consecuencia, más cercano es el equilibrio de mercado al equilibrio competitivo (ver por ejemplo Allaz y Vila, 1993; Green, 1999; Powell, 1993; Newbery, 1995; Scott, 1998, y Wolak, 2000) ${ }^{16}$. Este resultado se deriva del hecho de que mientras más contratado está un productor, menos dependen sus ingresos del precio en el mercado spot, por lo que el incentivo para presionar el alza de este precio también es menor (o nulo, en el margen). Hay ocasiones en que el incentivo opera incluso en la dirección contraria. Así por ejemplo, cuando el productor está sobrecontratado, es decir cuando sus compromisos contractuales superan su producción total, la posición del generador en el mercado spot es la de un comprador neto, por lo que tiene incentivo para presionar a la baja el precio.

${ }^{16}$ Este resultado contrasta con el de Liski y Montero (2005), quienes muestran que bajo ciertas circunstancias los contratos facilitan la colusión de los generadores. 
La evidencia en el caso de sistemas hidrotérmicos, como el chileno, es limitada. Así por ejemplo, Scott (1998) sólo estudia la relación entre el nivel de contratación y la generación total de las plantas hidráulicas. Tal análisis excluye el estudio de la decisión de asignación intertemporal del agua, por lo que no es posible hacer una aseveración definitiva en torno al ejercicio de poder de mercado

En la Tabla $\mathrm{N}^{\circ} 5$ se presentan los resultados de una simulación del equilibrio de mercado que se observaría si los generadores vendieran el $50 \%$ de su producción a través de contratos ${ }^{17}$. Se supone que los contratos son de tipo financiero, es decir, ambas partes realizan sus transacciones directamente en el mercado spot y se compensan mutuamente por cualquier diferencia entre el precio spot $(P)$ y el precio del contrato $(W)$. Así por ejemplo, si $P>W$, el productor paga directamente al consumidor $\$(P-W)$ por cada unidad contratada. La discusión en torno al incentivo para contratar y a la forma como se determina tanto el volumen como el precio al que se contrata se deja para más adelante.

\begin{tabular}{lccccccc}
\hline $\mathrm{t}$ & $\begin{array}{c}T_{E} \\
{[2]}\end{array}$ & $\begin{array}{c}H_{E} \\
{[3]}\end{array}$ & $\begin{array}{c}T_{G} \\
{[4]}\end{array}$ & $\begin{array}{c}T_{F} \\
{[5]}\end{array}$ & $\begin{array}{c}H_{F} \\
{[6]}\end{array}$ & $\begin{array}{c}\mathrm{Q} \\
{[7]}\end{array}$ & $\begin{array}{c}\text { Precio } \\
{[8]}\end{array}$ \\
\hline & & & & & & & \\
1 & 564,4 & $1.789,7$ & 950,6 & 556,7 & 417,3 & $4.672,7$ & 38,3 \\
2 & 564,4 & $1.669,3$ & 950,6 & 556,7 & 188,3 & $4.323,2$ & 37,4 \\
3 & 564,4 & $1.571,4$ & 950,6 & 556,7 & 146,5 & $4.183,6$ & 36,7 \\
4 & 564,4 & $1.273,3$ & 950,6 & 556,7 & 146,5 & $3.885,5$ & 34,6 \\
5 & 564,4 & 972,0 & 950,6 & 556,7 & 146,5 & $3.584,3$ & 32,2 \\
6 & 564,4 & 848,1 & 918,0 & 556,7 & 146,5 & $3.427,8$ & 30,0 \\
\hline
\end{tabular}

Donde $\quad \mathrm{T}_{\mathrm{i}}=$ producción térmica de la empresa “i” en la hora $t(\mathrm{MW})$;

$\mathrm{H}_{\mathrm{i}}=$ producción hidráulica de la empresa "i”" en la hora $t(\mathrm{MW})$;

$\mathrm{i}=\mathrm{E}$ (Endesa), G (Gener), F (pequeños productores);

$\mathrm{Q}=$ producción total en la hora $t(\mathrm{MW})$.

Resultado 3: La incorporación de los contratos reduce el incentivo para ejercer poder de mercado. Esto se traduce en precios y en decisiones de producción tanto estáticas como dinámicas más cercanas al equilibrio competitivo.

${ }^{17}$ Este $50 \%$ se calcula con respecto a la producción que se observaría si el equilibrio fuera competitivo. Esta forma de calcular el volumen contratado permite incorporar el hecho de que el monto contratado no necesariamente es constante a lo largo del mes. 
Mientras más contratados están los productores, menores son los precios y los márgenes que los productores cobran ${ }^{18}$. La Figura $\mathrm{N}^{\circ} 5$ muestra que la brecha entre el equilibrio competitivo y el equilibrio Cournot con $50 \%$ de contratos es bastante menor en relación con el equilibrio Cournot sin contratos. La brecha se reduce especialmente en los períodos de mayor demanda, que son precisamente aquellos en que se ejercía mayor poder de mercado en el caso base.

FIGURA N ${ }^{\circ}$ 5: $\quad$ EFECTO DE LOS CONTRATOS EN EL PRECIO DE EQUILIBRIO

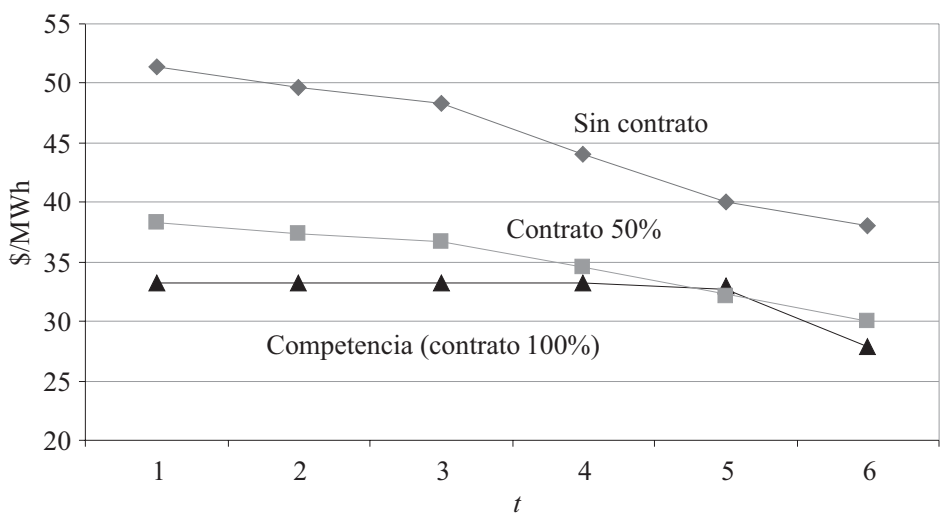

El mayor cambio en el comportamiento se observa en el caso de Endesa. Esta empresa no sólo aumenta en forma notoria su producción a partir de plantas térmicas, sino que además asigna proporcionalmente más agua a los períodos de mayor demanda y proporcionalmente menos a los períodos de menor demanda. La mayor eficiencia en la asignación intertemporal del agua mientras más contratadas están las empresas se refleja claramente en la Figura $N^{\circ} 6^{19}$.

${ }^{18}$ Cuando los generadores no venden a través de contratos, el equilibrio de mercado replica al equilibrio Cournot, mientras que cuando las empresas están totalmente contratadas, el equilibrio de mercado es prácticamente idéntico al equilibrio competitivo (no es exactamente igual, pues la producción en cada período es una variable endógena y luego es difícil de predecir con certeza al momento de firmar los contratos).

${ }^{19}$ Estos resultados difieren de los de Scott (1998), quien encuentra una relación positiva entre el nivel de contratos y la producción total a partir de plantas hidráulicas. Los resultados de esta simulación indican, en cambio, que la producción hidráulica total no cambia a consecuencia de los contratos; lo que cambia —en particular se torna más eficiente- es la asignación intertemporal del agua. 
FIGURA N N $^{\circ} \quad$ EFECTO DE LOS CONTRATOS EN LA ASIGNACIÓN INTERTEMPORAL DEL AGUA

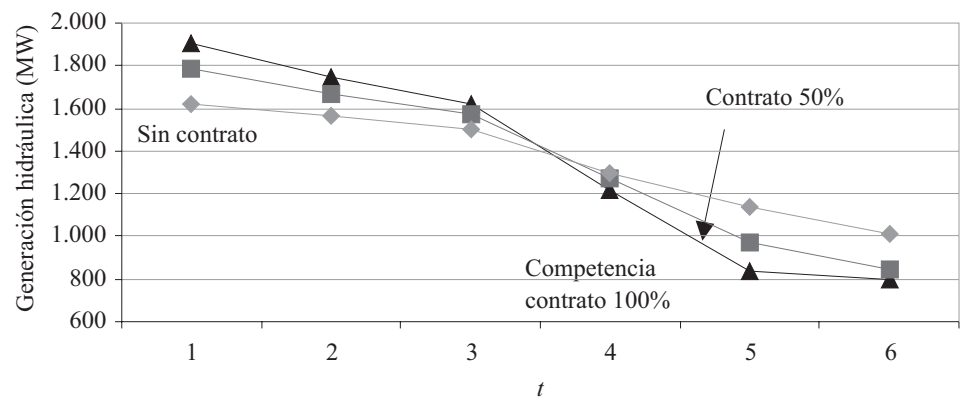

Estos resultados indican que la práctica de vender parte de la producción en un mercado de contratos es una medida efectiva en cuanto a mitigar el poder de mercado. De hecho, Endesa margina en promedio sólo el $56 \%$, por debajo del $71 \%$ observado anteriormente. Este resultado está en línea con la evidencia empírica (Newbery, 1997; Bushnell et al. 2004).

Finalmente cabe destacar que el desarrollo de un mercado de contratos influye en el incentivo para ejercer poder de mercado a través de otros mecanismos no considerados en el modelo usado para las simulaciones en este trabajo. Entre estos mecanismos se puede mencionar el efecto de los contratos en la entrada a la industria. Usualmente se argumenta que los contratos facilitan la entrada de nuevos competidores y de este modo contribuyen a moderar el comportamiento de los actuales actores de la industria. En la práctica, sin embargo, también podría ocurrir precisamente lo contrario. La dirección del efecto depende en forma crucial del mecanismo a través del cual se asignan los contratos a un determinado productor. Así por ejemplo, el plazo transcurrido entre la selección de un generador y el momento en que efectivamente se debe entregar el suministro es crucial en la posibilidad de que nuevos agentes puedan participar del proceso. Otros factores por considerar son el tamaño del bloque de energía que se contrata y el incentivo que tiene el comprador para contratar (esto se discute más adelante en forma detallada). Luego, si se pretende descansar en el mecanismo de contratos para aminorar el problema de poder de mercado es de extrema importancia el diseño de las bases de la licitación a través de la cual se asigna el contrato a una determinada empresa. Cualquier error podría resultar en la creación de una barrera de entrada a la industria, precisamente lo opuesto a lo que se pretendía conseguir. 


\subsection{Análisis comparativo de las medidas propuestas}

Estos resultados indican que las medidas propuestas contribuyen en mayor o en menor grado a mitigar el problema de poder de mercado. En términos cualitativos, está claro que permiten acercar el equilibrio al nivel competitivo y que las distorsiones tanto asignativas como productivas son menores. Dadas las formas funcionales supuestas, es posible comparar estas medidas en base a un indicador objetivo como es el efecto en bienestar, medido como la suma del excedente del productor y del consumidor. Los resultados se resumen en la Tabla $\mathrm{N}^{\circ} 6$.

\begin{tabular}{lrrrrrr}
\hline & Endesa & Gener & Otros* & Exc. Prod. & Exc. Cons & Exc. Total \\
& & & & & & \\
\hline & 118,8 & 172,1 & 159,5 & 137,1 & 72,6 & 94,9 \\
Duopolio Cournot & 90,2 & 109,6 & 118,2 & 114,0 & 91,0 & 99,0 \\
Vta. parque térmico & 31,2 & 106,2 & 115,9 & 112,0 & 92,4 & 99,2 \\
Vta. parque hidráulico & - & - & - & 109,4 & 93,9 & 99,3 \\
Contratos 50\% & 100,0 & 100,0 & 100,0 & 100,0 & 100,0 & 100,0 \\
Competencia & & & & & & \\
\hline
\end{tabular}

* Pequeños productores “originales”.

El primer resultado que llama la atención es que las tres medidas analizadas son relativamente similares en términos de bienestar, siendo marginalmente peor la enajenación del parque térmico. Las medidas que consideran la venta de una parte del parque generador de Endesa resultan en una pérdida directa para todos los productores, pues se puede ejercer menos poder de mercado. Obsérvese que si bien la posición de Gener se fortalece en términos relativos con cualquiera de estas medidas, aquélla prefiere que Endesa conserve todo su portfolio de generación, de modo que pueda empujar los precios al alza lo más posible. Por el mismo motivo, Gener y los pequeños productores prefieren que, en caso de decidir vender una parte de los activos, sean las plantas térmicas y no las hidráulicas las que se vendan, pues de ese modo Endesa mantendría el control de las plantas que le confieren el poder en el mercado ${ }^{20}$.

${ }^{20} \mathrm{Si}$ bien la posición de Endesa se ve considerablemente deteriorada, en la práctica esto no necesariamente ocurre en la magnitud indicada, pues Endesa recibirá ingresos por la venta de las plantas que deberá enajenar. 
No es posible analizar cuantitativamente el efecto de los contratos en los excedentes tanto del consumidor como del productor, pues ello requiere información en torno al precio de los contratos. A pesar de lo anterior, es posible hacer estimaciones a nivel agregado si suponemos que cualquier diferencia de precio entre el precio spot y el de los contratos origina sólo transferencias entre los agentes y ninguna pérdida de bienestar. En tal caso, la introducción de los contratos reduce el costo de bienestar desde $5,1 \%$ a menos de $1 \%$. Parte de esta ganancia en bienestar se explica porque no sólo la producción total es más cercana al nivel competitivo, sino porque además se reduce el costo que originaba el hecho de que plantas eficientes eran retenidas fuera del parque generador. Esto determina que el mayor costo de producción que resulta del despacho ineficiente sea menos del $8 \%$ (versus el $20 \%$ de mayor costo que resultaba en el equilibrio sin contratos).

Los resultados de las simulaciones realizadas son tentadores, pues muestran que existen medidas de política a las que la autoridad podría recurrir para complementar la liberalización del mercado spot y así mitigar cualquier problema de competencia al que esta reforma pudiera dar origen. Tal conclusión se debe contrastar sin embargo con el hecho de que ninguna de las medidas propuestas se puede implementar en forma simple, y que aun en tal caso pueden desarrollarse problemas de tipo práctico que hagan imposible su correcto funcionamiento. El problema más obvio es la factibilidad de obligar a Endesa a vender parte de su parque generador. Difícilmente esta empresa estará dispuesta a hacerlo en forma voluntaria, pues ello significaría perder su poder de mercado, el que además no es transferible a un posible comprador. Por otro lado, no está claro que la autoridad pueda forzarla a hacerlo sin violar el derecho de propiedad respectivo.

En el caso de los contratos, el problema es que para que esta medida efectivamente constituya un escudo de protección al problema de competencia en el mediano y largo plazo es necesario que se desarrolle un mercado de contratos voluntario suficientemente profundo, lo cual no es posible garantizar. En particular, antes de descansar en este mecanismo, la autoridad debería evaluar cuán probable es que los productores en realidad estén dispuestos a vender parte de su producción a través de contratos. Una vez que se toma en cuenta que al firmar contratos los productores están renunciando a su posibilidad de ejercer poder de mercado, no es posible afirmar con certeza que el mercado de contratos se vaya a desarrollar en forma voluntaria ${ }^{21}$.

${ }^{21}$ Harvey y Hogan (2000) desarrollan este argumento en forma extensa. 
En la literatura se han discutido al menos cuatro posibles motivos por los cuales se podría desarrollar el mercado de contratos. En primer lugar, Wolak (2000) y Allaz y Vila (1993) desarrollan modelos que les permiten argumentar que los productores venden contratos con el objeto de mejorar su situación en el mercado spot. Sus resultados sin embargo dependen de supuestos fuertes, como demanda por electricidad muy elástica, en el primer caso, y competencia Cournot en el segundo. Green (1999) y Powell (1993) argumentan que los productores podrían estar dispuestos a vender contratos aun anticipando que ello resultaría en una posición más débil en el mercado spot, si es que existen consumidores aversos al riesgo dispuestos a pagar una prima (i. e., precio contrato mayor que precio esperado en el mercado spot) que los proteja de tal riesgo. Powell (1993) enfatiza además que el mercado de contratos podría desarrollarse como resultado de un comportamiento estratégico por parte de los consumidores. Estos agentes están dispuestos a comprar contratos, aun pagando una prima, pues anticipan que de ese modo los productores tendrán menos incentivo para ejercer poder de mercado posteriormente en el mercado spot. Este resultado es válido incluso en presencia de consumidores neutros frente al riesgo. Finalmente Wolak (2000), Powell (1993) y Green (1999) han mostrado el rol que ha tenido la regulación en el desarrollo del mercado de contratos en Australia y en el Reino Unido. En ambos casos el precio de los contratos se encontraba sobre el precio spot.

Es difícil afirmar con certeza si las condiciones estarán dadas en la industria chilena para el desarrollo de un mercado de contratos. Lo único que se puede hacer en esta etapa es simplemente conjeturar. Es de esperar que la estrategia de la autoridad siga la aplicada en el extranjero y obligue a los productores a vender parte de su producción a través de contratos ${ }^{22}$. Esta alternativa sin embargo sólo posterga para el momento en que tales contratos expiren la interrogante en torno a si el mercado se desarrollará o no. La pregunta relevante es si llegado el momento los agentes tendrán el incentivo para vender o comprar contratos.

En el caso chileno, y dada la normativa actual, son dos los candidatos a contratar. Por un lado están los clientes libres y por otro las empresas de distribución. En el primer caso, actualmente ya opera un mercado de contratos no regulados aunque en forma limitada debido a ciertas deficiencias de la regulación. Estos consumidores usualmente se desenvuelven en otros sectores y contratan con el objetivo de estabilizar sus gastos por concepto de energía. Es de esperar que las modificaciones introducidas por

${ }^{22}$ El precio contratado posiblemente no tendrá efecto —en esta etapa - en el desarrollo del mercado de contratos, pero sí tendrá efectos distributivos. 
la llamada "ley corta”, en particular la reducción en el límite mínimo para pertenecer a esta categoría y la regulación de los peajes de distribución, resulten en una mayor profundización del mercado de contratos, al menos en este segmento.

El incentivo para contratar por parte de las distribuidoras es ciertamente menos claro. En la actualidad las distribuidoras deben comprar a través de contratos toda la demanda de sus consumidores regulados a un precio que después es traspasado directamente a la tarifa al usuario final

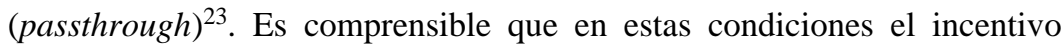
para contratar, y en particular el incentivo para negociar un buen precio sea bastante pequeño. Gran parte del problema viene dado por la existencia del passthrough del precio de generación. Este elemento produce ventajas y desventajas. Por un lado, permite que los consumidores enfrenten una señal de precio un poco más cercana al verdadero costo de producir electricidad. Por otro lado, tiene el problema de que la empresa distribuidora no gana nada con negociar un buen precio. Si esta situación no cambia, aun cuando el mercado de contratos podría desarrollarse, éste podría constituirse en la práctica en un mecanismo que facilite los comportamientos anticompetitivos en vez de dificultarlos. Así por ejemplo, podría dar lugar a que las empresas distribuidoras favorezcan a productores relacionados o bien que contraten a cambio de beneficios distintos del precio, como podría ser el no vender a los clientes libres localizados al interior de la zona de concesión.

\section{Conclusiones}

Este trabajo alerta sobre los peligros que conlleva la liberalización del mercado spot de la industria eléctrica en Chile. En particular, se muestra que esta reforma resultará en menor bienestar por dos motivos: ejercicio de poder de mercado y despacho ineficiente de las plantas. Los resultados de las simulaciones realizadas muestran que quien realmente estaría en posición de ejercer poder de mercado es Endesa. Tanto Gener como Colbún maximizan su utilidad comportándose como tomadores de precio la mayor parte del tiempo. La principal herramienta con que cuenta Endesa para influir en el precio de mercado es su parque hidráulico, específicamente las plantas asociadas a embalses con capacidad de regulación. La estrategia que utilizaría consiste en restringir la operación de su parque térmico al mínimo y distorsionar la asignación intertemporal del agua, asignando relati-

${ }^{23}$ Esto es cierto tanto antes como después de las modificaciones introducidas en la ley eléctrica en mayo de este año. 
vamente poca agua a períodos en que la demanda residual es menos elástica y relativamente mucha agua a períodos en que tal demanda es más elástica. Lo anterior determina que el despacho sea ineficiente aun restringiendo la operación de las plantas térmicas al volumen de producción total escogido por los productores Cournot, pues las plantas despachadas no son aquellas que minimizan el costo de operación del sistema ${ }^{24}$.

Posteriormente se analizan dos posibles medidas que se podrían implementar para reducir el problema de competencia: la enajenación de parte del parque generador de Endesa y la obligación de vender parte de la producción a través de contratos. Las simulaciones realizadas muestran que ambas medidas son efectivas en cuanto a acercar el equilibrio de mercado al competitivo, tanto a nivel asignativo como a nivel productivo. A pesar de lo anterior, no es clara la conveniencia de descansar en estas medidas, pues ambas adolecen de problemas serios. En el caso de la primera, hay dudas razonables en torno a la factibilidad de obligar a Endesa a vender parte de su parque generador, renunciando a través de ello a su poder de mercado. Por otro lado, si bien el desarrollo de un mercado de contratos puede constituir una adecuada protección frente a problemas de competencia en el mercado spot, tal desarrollo no está garantizado. Si la autoridad chilena desea confiar en este mecanismo, deberá ser extremadamente cuidadosa en los incentivos que las partes tienen para contratar en forma voluntaria. Descansar sólo en la regulación que obliga a los productores a contratar es una medida no sólo insuficiente sino también ingenua. Los contratos necesariamente expirarán en algún momento y en tal momento será la forma como esté regulada la industria la que proveerá los incentivos para contratar. Un último punto que debe tenerse en cuenta en relación con la importancia que tiene la correcta regulación de la industria y de cuánto se puede confiar efectivamente en el mercado de contratos es el resultado de Powell (1993) en torno a que mientras más oportunidades tienen los generadores para cooperar, menor será el volumen contratado.

${ }^{24} \mathrm{El}$ lector puede preguntarse legítimamente si el comportamiento estratégico que se anticipa en este trabajo, en el caso de que el mercado se liberalizara, lo podrían estar replicando hoy las decisiones de despacho del CDEC. Los mismos argumentos que se manejan para explicar por qué es poco probable que los generadores “inflen” los costos marginales explican por qué es poco probable que la asignación intertemporal del agua esté siendo distorsionada. Dado que el precio de venta de la energía, el precio de nudo, se calcula en forma independiente de los costos marginales de corto plazo efectivos, los generadores no tienen incentivo para coludirse y para distorsionar el uso del agua, pues ello redundaría en costos marginales más altos perjudicando a aquellos generadores en posición deficitaria que deban comprar energía en el mercado spot. Estos generadores tienen, pues, el incentivo correcto para vigilar que el despacho efectivamente apunte a la minimización de los costos. 
Un problema más de fondo se relaciona con la motivación para una reforma como la que aquí se analiza. El argumento usual es terminar con la regulación, pues es muy costosa y rígida, por lo que no siempre entrega señales adecuadas para la inversión y para el consumo. Paralelamente se espera descansar en el mercado, bajo el supuesto de que su funcionamiento resultará en un equilibrio competitivo, en menores distorsiones y de este modo en una maximización del bienestar social. Los resultados de este trabajo indican que este objetivo no se alcanza a través de la liberalización del mercado spot. De insistir en tal objetivo deberían implementarse medidas adicionales. La pregunta entonces es: ¿tiene sentido reemplazar la regulación actual por una reforma que pretende "dejar operar al mercado", pero que en la práctica requerirá de medidas regulatorias complementarias?

Si bien es cierto que ha llegado el momento de introducir cambios a la normativa actual, estos resultados indican que la liberalización del mercado spot ciertamente no es el camino que se debe seguir. Por lo menos mientras se mantengan las condiciones actuales.

\section{APÉNDICE}

En este Apéndice se presentan el modelo y los datos que se utilizaron para simular el equilibrio si el mercado spot fuera liberalizado.

\section{A.1. EL MODELO}

La actividad de generación es modelada como un duopolio Cournot que interactúa con pequeños productores sin poder de mercado. La presencia de recursos hidráulicos de embalse determina que las decisiones de producción que tomen los generadores tengan efecto no sólo en el período en que éstas se toman, sino en todo el horizonte de planificación. El modelo asume que los productores maximizan sus utilidades en un horizonte de planificación de un mes, el cual puede ser representado a través de seis subperíodos de una hora de duración cada uno, a los que se les designa como $t$. Estos subperíodos están ordenados de mayor a menor, de modo que $t=1$ indica el período de mayor demanda, y $t=6$ el de menor demanda de mercado.

Siguiendo a Arellano (2003), el problema de optimización que resuelve Endesa es:

$$
\max \sum_{t}^{6}\left\{P_{t}\left(q_{t}\right)\left(H_{E t}+T_{E t}\right)-C T_{E}\left(T_{E t}\right)\right\}
$$


sujeto a

$T_{E}{ }^{M I N} \leq T_{E t} \leq T_{E}{ }^{M A X} \forall t$ (restricción de mín./máx. cap. térmica)

$H_{E}{ }^{M I N} \leq H_{E t} \leq H_{E}{ }^{M A X} \forall t$ (restricción de mín./máx. cap. hidráulica)

$\sum_{t}^{6} H_{E t} \leq H_{E t}{ }^{\text {TOT }}$ (máxima disponibilidad de agua)

Gener resuelve un problema más simple:

$\max \sum_{t}^{6}\left\{P_{t}\left(q_{t}\right)\left(T_{G t}\right)-C T_{G}\left(T_{G t}\right)\right\}$

sujeto a

$T_{G}{ }^{M I N} \leq T_{G t} \leq T_{G}{ }^{M A X} \forall t$ (restricción de mín./máx. cap. térmica)

donde $P_{t}\left(q_{t}\right)$ es la función inversa de la demanda residual que enfrenta cada productor Cournot en el período $\mathrm{t}$ y $q_{t}$ es la producción total de las dos empresas en el período t. La producción de las plantas térmicas e hidráulicas de la empresa i se denotan como $T_{i}$ y $H_{i}$ respectivamente $\left(H_{G}=0\right)$. $C T_{i}\left(T_{i t}\right)$ es la función de costo total de la empresa $i$.

La optimización de los productores está sujeta a dos tipos de restricciones. Por un lado, la producción en cada período debe estar entre los límites establecidos por la capacidad mínima y máxima de la planta respectiva (ecuaciones 2, 3 y 6). Por otro lado, la ecuación 4 establece que en todo el horizonte de planificación se puede generar, como máximo, la cantidad de energía equivalente al agua almacenada en el embalse $\left(H_{E}^{T O T}\right)$. Obsérvese que el precio sombra de la restricción - constante a lo largo del horizonte de planificación - indica el valor marginal del agua (VMA), esto es, en cuánto aumentarían las utilidades de Endesa si el agua disponible para ser generada aumentara en una unidad (en términos de energía equivalente). Dado que el VMA indica cuánto estaría dispuesta a pagar Endesa por una unidad adicional de agua, este precio sombra puede ser interpretado también como el costo marginal del agua ${ }^{25}$.

La solución de este modelo indica que en cada subperíodo del horizonte de planificación cada productor elige su nivel de producción de modo

${ }^{25}$ En caso de que alguna de las restricciones de mínima y máxima capacidad sea activa, el costo marginal relevante debe incluir también el respectivo precio sombra. 
tal que el costo marginal de cada planta iguale al ingreso marginal. Cuando las restricciones de mínima y máxima capacidad no son activas, Endesa asigna su producción hidráulica de modo tal que el costo marginal de las plantas térmicas sea igual al VMA. La intuición que hay detrás de este resultado es simple: producir un kilowatt hora (kwh) adicional con agua desplaza la producción de un kwh de una central térmica, generando un ahorro dado por el costo marginal de esta planta. Este beneficio debe ser igual, en el margen, al costo implícito de tal decisión, dado por el menor ingreso que se obtendrá en un período siguiente cuando haya menos agua disponible para generar, dado por el VMA. Arellano (2004) muestra que en la práctica la estrategia de los productores Cournot se traduce en que el monopolista explota las diferencias en la elasticidad precio de la demanda residual entre períodos, asignando relativamente poca agua a los períodos en que la demanda es relativamente inelástica, y relativamente mucha a los períodos en que la demanda es más elástica, en comparación con la asignación que resultaría si el mercado fuera perfectamente competitivo. Muestra también que mientras mayor es la diferencia en la elasticidad precio entre períodos, mayor es el incentivo para ejercer poder de mercado.

Los pequeños productores resuelven exactamente el mismo problema que Endesa. A pesar de ello, el criterio que guía la decisión productiva difiere, pues estos productores no tienen poder de mercado y en consecuencia son tomadores de precio $\left(\frac{\partial P_{t}\left(q_{t}\right)}{\partial q_{t}}=0\right)$. En particular, la producción de cada planta/período se determina de modo tal que el costo marginal de la planta iguale al precio sombra del agua y al precio de mercado. Este resultado implica que en un escenario competitivo la asignación intertemporal del agua determina el costo marginal del sistema y, de este modo, el precio de equilibrio.

Las estrategias de producción y en particular la decisión del uso del agua disponible difieren entre el productor competitivo y el productor con poder de mercado, pues mientras Endesa asigna el agua de modo de igualar el ingreso marginal entre períodos, el comportamiento de los productores tomadores de precio apunta a igualar los precios. Como resultado, la diferencia de carga entre los períodos de punta y no punta se reduce cuando el agua se asigna en forma competitiva, mientras que aumenta cuando es un productor con poder de mercado quien toma tal decisión.

Este modelo no incorpora aspectos de competencia dinámica como la interacción repetida de los generadores, ni las características del sistema de transmisión, por lo que los resultados podrían subestimar el problema de poder de mercado. 
A continuación se modifica el modelo descrito en la sección anterior con el objeto de estudiar cuál es el efecto del nivel de contratación en el incentivo para ejercer poder de mercado y en la distorsión resultante en relación con el equilibrio competitivo. Para ello se supone que los generadores venden un porcentaje de su producción (“ $x$ ”) a través del mercado de contratos a un precio exógenamente definido $(W)$ y que cualquier diferencia entre el volumen contratado y la producción se transa en el mercado spot al precio $P$. Se supone que los contratos son de tipo financiero, es decir, ambas partes realizan sus transacciones directamente en el mercado spot y se compensan mutuamente por cualquier diferencia entre el precio spot y el precio del contrato. Así por ejemplo, si $P>W$, el productor paga directamente al consumidor $(P-W)$ por cada unidad contratada.

Para incorporar el efecto de los contratos, sólo es necesario modificar la función objetivo descrita anteriormente (ecuaciones y para el caso de Endesa y Gener respectivamente) de la siguiente manera:

$\max \sum_{t}^{6}\left\{P_{t}\left(q_{t}\right)\left(q_{i t}-k_{i t}\right)-C T_{i}\left(T_{i t}\right)+k_{i t} W_{i t}\right\} \quad i=E, G$

donde $q_{i t}$ es la producción total del productor i $\left(q_{i t}=H_{i t}+T_{i t}\right)$, $k_{i t}$ es el volumen contratado y $W_{i t}$ es el precio contratado. El último término corresponde al ingreso que obtiene el productor de las ventas en el mercado de contratos, por lo que no depende directamente del equilibrio en el mercado spot. La condición de primer orden está dada por la siguiente ecuación:

$$
\frac{\partial L}{\partial q_{i t}}=P_{t}\left(q_{t}\right)+\left(q_{i t}-k_{i t}\right) \frac{\partial P_{t}\left(q_{t}\right)}{\partial q_{i t}}-\frac{\partial C T_{i}\left(q_{i t}\right)}{\partial q_{i t}}=0 \forall t
$$

Esta ecuación difiere con respecto a la encontrada en el caso sin contratos en que el ingreso marginal que el productor iguala con el costo marginal sólo depende de aquella producción que el generador vende en el mercado spot $\left(q_{i t}-k_{i t}\right)$ y no de la producción total. Luego, mientras menor es la producción que se debe vender directamente en el mercado spot, más cercano es el ingreso marginal al precio y menor la diferencia entre éste y el costo marginal. 


\section{A.2. DAtos}

El equilibrio de mercado se simuló utilizando información de demanda y costos del Sistema Interconectado Central para el año 2002. Con el objetivo de simplificar la notación, sólo se utilizará el subíndice t cuando su omisión pueda conducir a error. La estimación se realizó usando información correspondiente al mes de abril, mes en que tradicionalmente se produce la demanda máxima del sistema.

\section{A.2.1. Función de costo marginal (plantas térmicas)}

El costo marginal de cada productor se construyó agregando los costos marginales de sus respectivas plantas estimados en base a información del CDEC-SIC. Se supone que éstos son constantes hasta su capacidad máxima.

La capacidad máxima de cada planta fue ajustada por un factor que incluye la disponibilidad media y el efecto de las pérdidas de transmisión y el consumo propio. En el caso de la primera variable, se utilizó el factor de indisponibilidad de punta acumulado al año 2002, mientras que en las otras dos variables se utilizó el promedio registrado en el período 1998-2002.

\section{A.2.2. Capacidad hidráulica}

Para simplificar la estimación del modelo, se asumió que tanto Endesa como los pequeños productores controlan sólo un embalse cada uno, cuya capacidad equivale a la suma de todos los embalses disponibles en la realidad. Se consideró además que aquellas centrales de pasada ubicadas aguas abajo de un embalse también pertenecen al sistema hidráulico respectivo, siendo éste el caso de las centrales Isla, Loma Alta y Rucúe. Luego, en el caso de Endesa, la capacidad hidráulica está determinada por la de las centrales Pangue (sistema Bío Bío), Canutillar (Chapo), Antuco y El Toro (Laja), Pehuenche, Cipreses, Curillinque, Isla y Loma Alta (Maule) y Rapel (Rapel). El parque hidráulico de los pequeños productores corresponde a las centrales Colbún, Machicura y San Ignacio.

$H_{E}^{T O T}$ se calculó como el promedio de la generación mensual efectivamente observada en las plantas hidráulicas en un año hidrológico normal de acuerdo a la Matriz de Energía proporcionada por el CDEC-SIC. Dado que esta información no existe para el Sistema Laja, se utilizó la generación promedio efectivamente registrada en el mes de abril de un año normal. Las 
restricciones de mínima y máxima capacidad $\left(H_{E}^{M I N}\right.$ y $\left.H_{E}^{M A X}\right)$ se estimaron en función de requisitos técnicos (30\% y 95\%, respectivamente) y de los contratos de riego, en caso de ser relevantes. Específicamente, el modelo asume que en abril del año 2002 hay 1.118 GWh disponibles para ser asignados, de los cuales el 87\% corresponde a la empresa Endesa.

\section{A.2.3. Demanda residual}

Los productores Cournot enfrentan una demanda residual dada por:

$$
D^{R}\left(P_{t}\right)=D_{t}\left(P_{t}\right)-\text { MRun }_{t}-T^{F}-H_{F t}
$$

donde $D_{t}\left(P_{t}\right)$ es la demanda de mercado, $M R u n_{t}$ es la generación obligada y $T^{F}$ y $H_{F t}$ indican la producción térmica e hidráulica de los pequeños productores, respectivamente. La estimación asume que estos productores producen al máximo de su capacidad térmica $\left(T^{F}=556,7 \mathrm{MW}\right)$, supuesto que no altera los resultados.

Demanda de mercado: Para diferenciar los períodos de demanda alta y baja, la curva de carga de abril de 2002 se dividió en seis períodos, llamando $t=1$ al período de mayor demanda y $t=6$ al período de menor demanda. La cantidad demandada en cada uno de ellos corresponde al promedio observado por hora en el período respectivo. Para cada subperíodo sólo existe una observación cantidad-precio, por lo que no es posible estimar una función de demanda de mercado para cada período en forma directa. El enfoque utilizado consistió en suponer una forma funcional y parametrizarla en base a datos de precio y cantidad consumida en el SIC y a un supuesto de elasticidad precio de la demanda. En particular, se utilizó el precio de nudo y se supuso que la demanda de mercado era lineal con forma $D_{t}\left(P_{t}\right)=A_{t}-B P_{t}$ con pendiente constante entre períodos. La demanda se determina de modo tal que la elasticidad precio $(\varepsilon)$ en el punto precio-cantidad observado sea igual a $-1 / 3^{26-27}$. Los valores de las constantes A y B utilizados para parametrizar la demanda se reportan en la Tabla $N^{\circ} 7$, columnas [4] y [5]. La

${ }^{26}$ Las estimaciones en torno a la elasticidad de la demanda difieren a lo largo de la literatura, y algunos resultados son muy dependientes del método y de la definición de corto y largo plazo utilizada. La estimación más reciente para el caso chileno fue realizada por Benavente et al. (2005). Ellos estiman que la elasticidad precio de la demanda residencial por energía eléctrica es $-0,0548$ en un mes y -0,39 en el largo plazo.

27 Estimaciones preliminares indican que las conclusiones no cambian si se usa el precio spot en vez del precio de nudo o bien si se asume una forma funcional diferente (demanda lineal no paralela entre períodos, o demanda con elasticidad constante). 
TABLA N ${ }^{\circ} 7: \quad$ PARAMETRIZACIÓN DE DEMANDA, ABRIL 2002, $\varepsilon=-1 / 3$

\begin{tabular}{|c|c|c|c|c|c|c|c|}
\hline \multirow{2}{*}{\multicolumn{2}{|c|}{$\begin{array}{c}\text { Carga promedio } \\
\text { por hora } \\
\text { (MW) }\end{array}$}} & \multirow{2}{*}{$\begin{array}{c}\text { Precio } \\
\text { (US\$/MWh) }\end{array}$} & \multicolumn{2}{|c|}{ Demanda de mercado } & \multirow{2}{*}{$\begin{array}{c}\varepsilon \\
\text { (Precio peak) }\end{array}$} & \multirow{2}{*}{$\begin{array}{l}\text { MRun } \\
\text { (MW) }\end{array}$} & \multirow{2}{*}{$\begin{array}{c}H_{F} \\
\text { (MW) }\end{array}$} \\
\hline & & & $\begin{array}{l}\text { Intercepto } \\
\text { (A) }\end{array}$ & $\begin{array}{l}\text { Pendiente } \\
\text { (B) }\end{array}$ & & & \\
\hline$t$ & [2] & [3] & [4] & [5] & [6] & [7] & [8] \\
\hline 1 & $4.834,6$ & 34,8 & $6.446,1$ & 46,3 & 0,33 & 394,0 & 417,3 \\
\hline 2 & $4.445,0$ & 34,8 & $6.056,5$ & 46,3 & 0,36 & 394,0 & 188,3 \\
\hline 3 & $4.272,6$ & 34,8 & $5.884,2$ & 46,3 & 0,38 & 394,0 & 146,5 \\
\hline 4 & $3.875,2$ & 34,8 & $5.486,7$ & 46,3 & 0,42 & 394,0 & 146,5 \\
\hline 5 & $3.465,5$ & 34,8 & $5.077,0$ & 46,3 & 0,47 & 394,0 & 146,5 \\
\hline 6 & $3.204,1$ & 34,8 & $4.815,6$ & 46,3 & 0,50 & 394,0 & 146,5 \\
\hline
\end{tabular}

columna [6] muestra que la combinación de los supuestos de demanda lineal y pendiente constante entre períodos resulta en que la demanda de mercado en horas peak es menos elástica que la demanda en las horas no peak (al mismo precio).

Generación obligada (MRun): Corresponde a la producción de aquellas plantas que no pueden ser utilizadas en forma estratégica. MRun se calculó en base a la generación promedio por hora observada en el caso de las dos pequeñas plantas térmicas cogeneradoras y a la generación promedio por hora que indica la Matriz de Energía en el caso de aquellas centrales hidráulicas de pasada (no asociadas aguas arriba con un embalse). Como indica la Tabla 7, columna [7], es constante a lo largo de los subperíodos. Dado que estas plantas no pueden ser usadas en forma estratégica, también fueron excluidas de las respectivas funciones de costo marginal.

Generación hidráulica de los pequeños productores $\left(H_{F}\right)$ : La producción hidráulica de los pequeños productores se asigna en base al criterio de peak shaving que resulta al resolver el respectivo problema de optimización. Esto es, el agua disponible se asigna entre períodos intentando igualar los precios entre períodos, o lo que es equivalente (considerando los supuestos de demanda que hemos utilizado) con el objetivo de eliminar los peaks asignando la mayor cantidad de agua posible — dadas las restricciones de mínima y máxima capacidad - a los períodos de mayor demanda. $H_{F}$ corresponde a la generación promedio por hora que se asigna a cada subperíodo y es reportada en la Tabla $N^{\circ} 7$, columna [8]. En la Figura $N^{\circ} 4$ se 
observa que la generación hidráulica de los pequeños productores es tan pequeña en relación con la demanda del sistema, que no es capaz de eliminar los peaks, preservando prácticamente completa la forma de la Curva de Carga.

\section{REFERENCIAS}

Allaz, B. y J. L. Vila: "Cournot Competition, Forward Markets and Efficiency". En Journal of Economic Theory, 59, 1-16 (1993).

Arellano, M. Soledad: “Three Essays on Market Power in Chile's Electricity Industry”. Ph. D. Thesis, MIT, 2003.

Arellano, M. Soledad: “Market Power in Mixed Hydro-Thermal Electric Industries". Documento de Trabajo Centro de Economía Aplicada $N^{\circ}$ 187, Departamento de Ingeniería Industrial, U. de Chile, 2004.

Arellano, M. Soledad y Pablo Serra: "Market Power in Price-regulated Power Industries". Mimeo, 2005.

Benavente, José Miguel, Alexander Galetovic y Ricardo Sanhueza: "Estimando la Demanda Residencial por Electricidad en Chile: El Consumo es Sensible al Precio". Cuadernos de Economía, Vol. 42, 31-62 (mayo 2005).

Borenstein, Severin y James Bushnell: "An Empirical Analysis of the Potential for Market Power in California's Electricity Market”. En Journal of Industrial Economics, 47, No 3, September 1999.

Borenstein, Severin, James Bushnell y Frank Wolak: "Measuring Market Inefficiencies in California's Wholesale Electricity Industry”. En American Economic Review, 92 (December, 2002) .

Bushnell, James, Erin Mansur y Celeste Saravia: "Market Structure and Competition: A Cross-Market Analysis of U.S. Electricity Deregulation”. CSEM Working Paper, 126, 2004.

ENRE: "International Seminar on Restructuring and Regulation of the Electric Power Sector”. Seminario realizado en noviembre 1995. Buenos Aires, 1997.

Green, Richard: "The Electricity Contract Market in England and Wales". En The Journal of Industrial Economics. Volume XLVII, No 1 (March) 1999a.

Harvey, Scott y William Hogan: "California Electricity Prices and Forward Market Hedging”. Mimeo, octubre 2000.

Joskow, Paul: "Lessons Learned from Electricity Liberalization in the UK and US". 2002. Disponible en web.mit.edu/pjoskow/www.

Joskow, Paul y Edward Kahn: "A Quantitative Analysis of Pricing Behavior In California’s Wholesale Electricity Market During Summer 2000”. En The Energy Journal, Vol. 23, No 4, 2002.

Liski, Matti y Juan Pablo Montero: "Forward Trading and Collusion in Oligopoly". En Journal of Economic Theory, 2005.

Newbery, David: "Power Markets and Market Power”. En The Energy Journal, Vol. 16, $\mathrm{N}^{\circ}$ 3, 1995.

Newbery, David: "Regulation of the Electricity Sector: Comments on Some Alternative Models”. En ENRE: International Seminar on Restructuring and Regulation of 
the Electric Power Sector. Seminario efectuado en noviembre 1995, Buenos Aires, y publicado en 1997.

Powell, Andrew: "Trading Forward in an Imperfect Market: The Case of Electricity in Britain”. En The Economic Journal, 103 (March 1993).

Scott, Tristam: "Hydro Reservoir Management for an Electricity Market with Long Term Contracts”. Thesis, University of Canterbury, 1998.

Wolak, Frank: "Market Design and Price Behavior in Restructured Electricity Markets: An International Comparison”. En Takatoshi Ito y Anne Krueger (editors), Competition Policy in the Asia Pacific Region, EASE. Volume 8. University of Chicago Press, 1999.

Wolak, Frank: "An Empirical Analysis of the Impact of Hedge Contracts on Bidding Behavior in a Competitive Electricity Market”. En International Economic Journal 14 (2), 2000. 